\title{
Genome-wide evidence for positive selection and recombination in Actinobacillus pleuropneumoniae
}

\author{
Zhuofei Xu, Huanchun Chen ${ }^{*}$ and Rui Zhou*
}

\begin{abstract}
Background: Actinobacillus pleuropneumoniae is an economically important animal pathogen that causes contagious pleuropneumonia in pigs. Currently, the molecular evolutionary trajectories for this pathogenic bacterium remain to require a better elucidation under the help of comparative genomics data. For this reason, we employed a comparative phylogenomic approach to obtain a comprehensive understanding of roles of natural selective pressure and homologous recombination during adaptation of this pathogen to its swine host.

Results: In this study, 12 A. pleuropneumoniae genomes were used to carry out a phylogenomic analyses. We identified 1,587 orthologous core genes as an initial data set for the estimation of genetic recombination and positive selection. Based on the analyses of four recombination tests, $23 \%$ of the core genome of A. pleuropneumoniae showed strong signals for intragenic homologous recombination. Furthermore, the selection analyses indicated that 57 genes were undergoing significant positive selection. Extensive function properties underlying these positively selected genes demonstrated that genes coding for products relevant to bacterial surface structures and pathogenesis are prone to natural selective pressure, presumably due to their potential roles in the avoidance of the porcine immune system.

Conclusions: Overall, substantial genetic evidence was shown to indicate that recombination and positive selection indeed play a crucial role in the adaptive evolution of A. pleuropneumoniae. The genome-wide profile of positively selected genes and/or amino acid residues will provide valuable targets for further research into the mechanisms of immune evasion and host-pathogen interactions for this serious swine pathogen.
\end{abstract}

\section{Background}

In the evolutionary history of many microorganisms, positive selection and homologous recombination are two indispensable driving forces for adaptation to new niches. Both of them contribute to the genetic variations that might influence the population diversification and adaptation of pathogenic microorganisms [1,2]. Recent studies on the genome-wide evolutionary dynamics have highlighted the important roles of selection and recombination in the molecular evolution of bacterial pathogens, including Escherichia coli [1], Listeria monocytogenes [3], Salmonella spp. [4], Streptococcus spp. [5], and Campylobacter spp. [6]. These analyses have revealed that a certain number of protein-coding genes subject to natural selection pressure are usually involved in the dynamical interactions between host and pathogen, especially in the

\footnotetext{
* Correspondence: chenhch@mail.hzau.edu.cn; rzhou@mail.hzau.edu.cn Division of Animal Infectious Disease, State Key Laboratory of Agricultural Microbiology, College of Veterinary Medicine, Huazhong Agricultural University, Wuhan 430070, China
}

() 2011 Xu et al; licensee BioMed Central Ltd. This is an Open Access article distributed under the terms of the Creative Commons Attribution License (http://creativecommons.org/licenses/by/2.0), which permits unrestricted use, distribution, and reproduction in any medium, provided the original work is properly cited. immune and defense-associated functions [1]. Diversifying selection operating on these genes may be caused by pathogen-host co-evolutionary arms race $[7,8]$.

In the present study, $d_{\mathrm{N}} / d_{\mathrm{S}}$-based methods were applied to detect evidence of genome-wide positive Darwinian selection. Estimating the ratio $(\omega)$ of the rate of nonsynonymous nucleotide substitutions $\left(d_{\mathrm{N}}\right)$ to that of synonymous substitutions $\left(d_{\mathrm{S}}\right)$ is a powerful approach for measuring selective pressure on the protein-coding level: $\omega=1,<1,>1$ indicate neutral evolution, purifying (negative) selection, and positive (adaptive) selection, respectively $[9,10]$. The codon models further developed by Nielsen and Yang allow variation in $\omega$ among sites [11], which have an extensive capability to find evidence for adaptive evolution in most functional genes where only a small fraction of amino acid sites are subject to strong positive selective pressure [12]. Thus far this approach has been widely used for genome-wide selection analyses in pathogenic viruses, bacteria, and eukaryotes $[9,13]$. A substantial number of genes encoding highly variable 
antigens are identified to undergo adaptive selection particularly on some functional sites for evasion of host immunity $[1,4,14]$.

Actinobacillus pleuropneumoniae, a Gram-negative coccobacillus belonging to the Actinobacillus genus of Pasteurellaceae family, is a strictly swine pathogen and colonizes in the upper respiratory tract of porcine [15]. This pathogen has caused an economically severe disease characterized by pulmonary lesions, pleuritis, and pericarditis in pigs [16]. According to the differences in capsular polysaccharides, $A$. pleuropneumoniae has been divided into 15 serovars [17]. The recent comparative genomics studies through both high-throughput approaches of genome sequencing and microarray have depicted the compositions of the pan-genome and confirmed the contribution of genes loss or gain to the diversity in virulence and serovar of $A$. pleuropneumoniae $[18,19]$. However, besides large genetic variations resulting from DNA acquisition and genome reduction, small sequence differences occurring in the conserved genes, including point mutations, insertions/deletions (indels), and intragenic recombination, may also play a crucial part in the alteration of antibiotic resistance, pathogenicity and immunogenicity $[20,21]$. But to date, no research pays enough attention to the linkages between genetic alterations and putative functional roles in intraspecies conserved genes of $A$. pleuropneumoniae at the whole genome level.

In order to further trace evolutionary trajectories on the core genome of pathogenic bacterium A. pleuropneumoniae, we employed a genome-wide analyses approach to investigate the effects of natural selection and homologous recombination operating on the coding genes. Our analyses focused on the evolutionary characterizations of core genome genes that are shared by $12 \mathrm{~A}$. pleuropneumoniae genomes. Many genes were shown to be under strong positive selective pressure and primarily associated with the fitness and immunogenic properties of this swine pathogen.

\section{Methods}

\section{Genome dataset and alignment}

Twelve genome sequences of $A$. pleuropneumoniae were retrieved from NCBI Genome database (http://www.ncbi. nlm.nih.gov/genome). The sequences included 3 complete genomes and 9 draft genome assemblies (see details in Table 1). Orthologous gene content information and annotation with COG functional classification have been defined in our recent work and used here [19]. To increase accuracy and power of selection analyses, an ortholog set was excluded if it satisfied any of the following criteria: the length of any gene lower than $80 \%$ of the maximum length, more than one gene from each genome or less than four sequences. Protein-coding sequences longer than 50 codons were used in this study. Subsequently, the orthologous protein sequences were aligned using a progressive method implemented in $\mathrm{T}$-Coffee v8.93 [22].

Frameshift mutations (indels of a number of nucleotides not divisible by three) can lead to high nonsynonymous substitution rates, resulting in more false positive results when positive selection was estimated based on $d_{\mathrm{N}} / d_{\mathrm{S}}$ ratios [5]. To avoid incorrect indels in the codon alignments, multiple sequence alignments were initially performed with amino acid sequences from each gene cluster, followed by conversion to the corresponding codon alignments using custom Perl scripts. The coding sequences located at the beginning or end of the contigs appeared to be more prone to frameshift sequencing errors. Therefore, we further assessed the quality for each alignment through obtaining the following information: overall identity, and identity in the first $30 \mathrm{nt}$ and last $30 \mathrm{nt}$ per alignment. The codon alignment sequences that contain frameshift mutations were checked and edited manually in the software MEGA4 [23] if identity is low.

\section{Calculation of $d_{N}, d_{\mathrm{S}}$, codon bias, nucleotide diversity and informative sites}

According to the method as defined by Nei and Gojobori [24], the number of synonymous nucleotide substitutions per synonymous site $\left(d_{\mathrm{S}}\right)$ and the number of nonsynonymous nucleotide substitutions per nonsynonymous site $\left(d_{\mathrm{N}}\right)$ were estimated for the resulting gene alignments using the program SNAP [25]. Gene-by-gene number of informative sites and genetic diversity were obtained from the output of the PhiPack program [26]. The analyses for the codon usage variation was performed by computing the effective number of codons $\left(\mathrm{N}_{\mathrm{c}}\right)$, which is a general measure of bias from equal codon usage in a gene. The $\mathrm{N}_{\mathrm{c}}$ value ranges from 20 for the strongest bias (where only one codon is used for each amino acid) to 61 for no bias $[27,28]$. The calculation of $\mathrm{N}_{\mathrm{c}}$ were implemented in the program CodonW 1.4 (http://codonw. sourceforge.net/).

\section{Detection of recombination events}

Since recombining fragments among aligned codon sequences have a profound effect on the detection of the positively selective evidence [29], we first tested for recombination signals between sequences in the alignment of orthologous genes. Four statistical procedures GENECONV [30], pairwise homoplasy index (PHI) [26], maximum $\chi^{2}$ [31] and neighbor similarity score (NSS) [32] were applied to discover the homologous recombination signals. Besides GENECONV version 1.81, the other three methods were implemented in the PhiPack package [26]. For the analyses of GENECONV, the parameter g-scale was set to 1 , which allows mismatches 
Table 1 Genome sequences of $A$. pleuropneumoniae used in this study

\begin{tabular}{|c|c|c|c|c|}
\hline Strain & GenBank accession no. & Genome size (Mbp) & No. of CDS (> 49 codons) & Reference \\
\hline JL03 & CP000687 & 2.24 & $2,101(2,035)$ & {$[60]$} \\
\hline L20 & СР000569 & 2.27 & $2,137(2,072)$ & [61] \\
\hline Ap76 & СР001091 & 2.33 & $2,203(2,134)$ & \\
\hline 4074 & ADOD00000000 & 2.26 & $2,180(2,101)$ & [19] \\
\hline S1536 & ADOE00000000 & 2.22 & $2,137(2,061)$ & [19] \\
\hline M62 & ADOF00000000 & 2.27 & $2,223(2,151)$ & [19] \\
\hline Fem $\phi$ & ADOG00000000 & 2.31 & $2,219(2,138)$ & [19] \\
\hline CVJ13261 & ADOI00000000 & 2.26 & $2,204(2,124)$ & [19] \\
\hline D13039 & ADOJ00000000 & 2.27 & $2,168(2,091)$ & [19] \\
\hline 56153 & ADOK00000000 & 2.27 & $2,195(2,120)$ & [19] \\
\hline 1096 & ADOL00000000 & 2.19 & $2,096(2,033)$ & [19] \\
\hline N273 & ADOM00000000 & 2.25 & $2,149(2,086)$ & [19] \\
\hline
\end{tabular}

within a recombining fragment. The $p$-values for inner fragments using 10,000 random permutations were used to indicate the significance of putative recombinant regions. For maximum $\chi^{2}$, a fixed window-size of $2 / 3$ the number of polymorphic sites was used. For PHI, the window size was set to 100 nucleotides. Simulated $p$-values were estimated based on 1,000 permutations for PHI, maximum $\chi^{2}$ and NSS.

\section{Detection of Selection}

Maximum likelihood (ML) phylogenetic trees were reconstructed for each gene in the dataset of the core genome genes using the PhyML program [33]. A general time-reversible (GTR) model of nucleotide substitution with the ML estimates for gamma distributed rate heterogeneity of four categories $\left(\Gamma_{4}\right)$ and a proportion of invariable sites were used in all tree reconstruction methods. The resulting topologies of ML trees were applied to the subsequent selection analyses.

To detect selective pressure acting on each coding gene, the rates of synonymous and nonsynonymous substitutions were estimated site-by-site using the codeml program from the PAML 4.2b package [34]. According to the topology of the resulting ML tree per gene alignment, two site-specific models that allow variable nonsynonymous $\left(d_{\mathrm{N}}\right)$ and synonymous $\left(d_{\mathrm{S}}\right)$ rate ratios $(\omega=$ $d_{\mathrm{N}} / d_{\mathrm{S}}$ ) among codons were applied to analyze our data set: M1a (NearlyNeutral) and M2a (PositiveSelection). Null hypothesis model M1a was nested with alternative selection model M2a. The latter model adds an extra site class for a fraction of positively selected amino acid sites with $\omega>1$; whereas models M1a only allows site classes with $\omega$ varying between 0 and 1 [10,35]. A likelihood ratio test (LRT) was carried out to infer the occurrence of sites subject to positive selective pressure through comparing M1a against M2a. Three replicates were run with codeml and the maximum likelihood values for each model were used in the LRT. The LRT statistic (twice the log-likelihood difference between the null and the alternative models) was compared with the $\chi^{2}$ distribution with two degrees of freedom. The Bayes empirical Bayes approach was employed to identify positively selected sites under the likelihood framework [36].

\section{Mapping of positively selected sites to structure models of proteins}

The web server PSORTb v3.0 was used to predict bacterial protein subcellular localization [37]. Integral betabarrel outer membrane proteins were predicted by BOMP [38]. The three dimensional structure model of the protein encoded by the gene that showed evidence for positive Darwinian selection was modeled using the Phyre server [39]. The sites subject to positive selective pressure were mapped onto the structure and visualized by PyMol (http://www.pymol.org/).

\section{Statistical analyses}

Multiple testing correction was performed to control for Type I errors according to the approach presented by Benjamini \& Hochberg [40]. For all genes tested for recombination and positive selection, $q$-values were calculated from $p$-values using the $\mathrm{R}$ package qvalue with the proportion of true null hypothesis set to $1\left(\pi_{0}=1\right)$ [41]. A false discovery rate (FDR) of $10 \%$ was used for the recombination analyses. As the tests used for detecting positive selection was conservative [42], an FDR of $20 \%$ was set.

The non-parametric Mann-Whitney U-test was employed to determine the significance level for the differences among the selected continuous variables (i.e., $d_{\mathrm{N}}, d_{\mathrm{S}}$, codon bias and nucleotide diversity) between a given COG functional categories and all other categories. Binomial test was used to estimate association between each COG category and evolutionary forces (i.e. positive selection and/or homologous recombination); Bonferroni corrections for multiple comparisons 
were performed according to the number of one-sided tests. The significance level was set to $5 \%$ in this study. All statistic analyses were carried out using Perl scripts and R 2.11.1 [43].

\section{Results}

Properties of orthologous genes in $12 \mathrm{~A}$. pleuropneumoniae genomes

In our recent work [19], 2,531 orthologous genes and 772 strain-specific genes have been identified in the pan-genome of 12 A. pleuropneumoniae strains using BlastClust. The above data set was used to further decode phylogenomic characterizations of pathogenic A. pleuropneumoniae. The evidence for homologous recombination and natural selection pressure whether operate on the conserved coding genes was estimated at the present study. After manually editing the aligned gene sequences and removing the low quality ones, a data set of sequence alignments of 1,960 orthologs was selected out, $81 \%(\mathrm{n}=$ $1,587)$ of which were core genes that are present one copy per genome and the remaining $(n=373)$ were distributed genes present in at least four genomes.

The codon bias for each orthologous gene was measured by the effective number of codons $\left(\mathrm{N}_{\mathrm{c}}\right.$ value $)$ calculated by Codon W [28]. The reduction in $\mathrm{N}_{\mathrm{c}}$ indicates strong bias that significantly correlates with high gene expressivity [44]. A. pleuropneumoniae genes in the COG functional categories "Energy production and conversion", "Translation", "Amino acid transport and metabolism", "Nucleotide transport and metabolism", and "Carbohydrate transport and metabolism" were evident to have higher codon usage bias $(P<0.001, P<0.001$, $P=0.003, P=0.001$, and $P<0.001$, respectively; onetailed U-test) compared with genes in other COG categories. As is well known, genes bearing stronger codon bias are likely to be highly expressed and have housekeeping features $[3,45]$. So, high codon bias of genes present in the five COG categories is likely to elucidate the necessity of relevant coding products for implementing fundamental life cycle and essential physiological activities of A. pleuropneumoniae.

A. pleuropneumoniae genes in the functional categories "Replication, recombination and repair" and "Amino acid transport and metabolism" represented a tendency to have higher rates of synonymous $\left(d_{\mathrm{S}}\right)$ nucleotide substitutions $(P=0.006$ and $P<0.001$, respectively; one-tailed U-test) in comparison with genes in other role categories (Table 2). On the other hand, genes in the functional categories "Replication, recombination and repair", "Amino acid transport and metabolism", "Coenzyme transport and metabolism" and "General functional prediction" showed a tendency to have higher rates of nonsynonymous $\left(d_{\mathrm{N}}\right)$ substitutions $(P=0.012, P=0.001, P=0.007$, and $P=0.007$, respectively; one-tailed U-test) in comparison with genes in other COG categories (Table 2). Positive correlation was observed between $d_{\mathrm{S}}$ and $d_{\mathrm{N}}$ values for each COG category of $A$. pleuropneumoniae genes, indicating that natural selection might uniformly act on synonymous and nonsynonymous sites per gene. In addition, it was worth noting that the average $d_{\mathrm{S}}$ and $d_{\mathrm{N}}$ values were significantly lower $(P=0.001$ and $P<0.001$, respectively; one-tailed U-test) for genes in the COGs "Translation" than for genes in other COG categories. It has been suggested that genes involved in the translation machinery, e.g. ribosomal proteins and tRNA synthetases, usually evolved slowly with low $d_{\mathrm{S}}$ and $d_{\mathrm{N}}$, probably due to structural and functional constraints required by the fundamental cell life cycle $[20,46,47]$.

\section{A substantial number of genes showing evidence for recombination in the core genome of $A$. pleuropneumoniae}

Among the 1,587 orthologous core genes, 2\% (29 genes) had no occurrence of nucleotide substitutions and thus were not further investigated for evidence of homologous recombination. Furthermore, among the remaining genes, 197 gene alignments that contain few informative sites less than two could not be analyzed with programs in PhiPack and were removed from the ortholog sets. Finally, $86 \%$ of total core genes were selected to conduct the subsequent recombination analyses through four approaches. The evolutionarily conserved core genes ( $\mathrm{n}=226)$ were summarized (Additional file 1) and the biological functions carried out by their coding products may be essential for the survival of $A$. pleuropneumoniae. Notably, conserved genes were significantly enriched in the COG category "Translation" with a low Bonferroni corrected $p$-value $(P<0.001$; Binomial test); this result was consistent with low $d_{\mathrm{S}}$ and $d_{\mathrm{N}}$ values mentioned before. These translation-associated proteincoding genes are generally involved in the fundamental cellular activity and thus hardly have any changes at the amino acid level as a result of functional constraints. Overall, among 12 A. pleuropneumoniae genomes, 822 orthologous core genes ( $52 \%$ of all 1,587 core genome genes) were identified to have significant evidence for recombination $(\mathrm{FDR}<10 \%)$ that was detected by at least one of the four tests (Additional file 2). A total of 493, 675, 659 and 559 orthologs were identified to have recombination signals using GENECONV, Maximum $\chi^{2}$, NSS and PHI, respectively. Additionally, a total of 149 , 148,160 , and 365 orthologs exhibiting recombination signals were identified by using one, two, three, and all four recombination tests, respectively.

It is worth noting that $23 \%$ of all core genes, which were selected as recombinants by all four methods for testing recombination, have more informative sites $(P<0.001$; 
Table 2 The rates of synonymous $\left(d_{S}\right)$ and nonsynonymous $\left(d_{N}\right)$ nucleotide substitutions among different functional categories for A. pleuropneumoniae genes

\begin{tabular}{|c|c|c|c|c|}
\hline COG category ${ }^{\mathrm{a}}$ & Number of genes analyzed & $d_{\mathrm{S}}( \pm \mathrm{se})^{\mathrm{b}}$ & $d_{\mathrm{N}}( \pm \mathrm{se})^{\mathrm{c}}$ & $r^{d}$ \\
\hline Energy production and conversion & 114 & $44.2 \times 10^{-3}\left( \pm 5.2 \times 10^{-3}\right)$ & $2.7 \times 10^{-3}\left( \pm 0.5 \times 10^{-3}\right)$ & 0.78 \\
\hline Cell cycle control and cell division & 26 & $59.7 \times 10^{-3}\left( \pm 13.0 \times 10^{-3}\right)$ & $3.3 \times 10^{-3}\left( \pm 0.8 \times 10^{-3}\right)$ & 0.69 \\
\hline Amino acid transport and metabolism & 133 & $80.1 \times 10^{-3}\left( \pm 6.6 \times 10^{-3}\right)$ & $4.1 \times 10^{-3}\left( \pm 0.4 \times 10^{-3}\right)$ & 0.80 \\
\hline Nucleotide transport and metabolism & 52 & $53.7 \times 10^{-3}\left( \pm 8.3 \times 10^{-3}\right)$ & $2.5 \times 10^{-3}\left( \pm 0.4 \times 10^{-3}\right)$ & 0.67 \\
\hline Carbohydrate transport and metabolism & 109 & $56.8 \times 10^{-3}\left( \pm 6.5 \times 10^{-3}\right)$ & $3.2 \times 10^{-3}\left( \pm 0.6 \times 10^{-3}\right)$ & 0.73 \\
\hline Coenzyme transport and metabolism & 92 & $59.5 \times 10^{-3}\left( \pm 7.7 \times 10^{-3}\right)$ & $4.7 \times 10^{-3}\left( \pm 0.7 \times 10^{-3}\right)$ & 0.57 \\
\hline Lipid transport and metabolism & 32 & $61.4 \times 10^{-3}\left( \pm 11.3 \times 10^{-3}\right)$ & $3.7 \times 10^{-3}\left( \pm 0.9 \times 10^{-3}\right)$ & 0.75 \\
\hline Translation & 151 & $42.9 \times 10^{-3}\left( \pm 4.5 \times 10^{-3}\right)$ & $2.1 \times 10^{-3}\left( \pm 0.3 \times 10^{-3}\right)$ & 0.58 \\
\hline Transcription & 78 & $69.4 \times 10^{-3}\left( \pm 11.8 \times 10^{-3}\right)$ & $3.8 \times 10^{-3}\left( \pm 0.8 \times 10^{-3}\right)$ & 0.86 \\
\hline Replication, recombination and repair & 94 & $83.3 \times 10^{-3}\left( \pm 11.8 \times 10^{-3}\right)$ & $6.2 \times 10^{-3}\left( \pm 1.3 \times 10^{-3}\right)$ & 0.69 \\
\hline Cell membrane and envelope biogenesis & 120 & $63.3 \times 10^{-3}\left( \pm 10.8 \times 10^{-3}\right)$ & $4.8 \times 10^{-3}\left( \pm 0.8 \times 10^{-3}\right)$ & 0.85 \\
\hline $\begin{array}{l}\text { Posttranslational modification, protein } \\
\text { turnover, chaperones }\end{array}$ & 84 & $55.3 \times 10^{-3}\left( \pm 7.2 \times 10^{-3}\right)$ & $3.6 \times 10^{-3}\left( \pm 0.9 \times 10^{-3}\right)$ & 0.75 \\
\hline Inorganic ion transport and metabolism & 124 & $63.5 \times 10^{-3}\left( \pm 6.1 \times 10^{-3}\right)$ & $3.9 \times 10^{-3}\left( \pm 0.4 \times 10^{-3}\right)$ & 0.67 \\
\hline $\begin{array}{l}\text { Secondary metabolites biosynthesis, } \\
\text { transport and catabolism }\end{array}$ & 12 & $34.3 \times 10^{-3}\left( \pm 11.4 \times 10^{-3}\right)$ & $2.7 \times 10^{-3}\left( \pm 0.9 \times 10^{-3}\right)$ & 0.90 \\
\hline General function prediction only & 203 & $65.7 \times 10^{-3}\left( \pm 6.1 \times 10^{-3}\right)$ & $4.6 \times 10^{-3}\left( \pm 0.5 \times 10^{-3}\right)$ & 0.82 \\
\hline Function unknown & 177 & $73.6 \times 10^{-3}\left( \pm 9.1 \times 10^{-3}\right)$ & $4.7 \times 10^{-3}\left( \pm 0.6 \times 10^{-3}\right)$ & 0.86 \\
\hline Signal transduction mechanisms & 30 & $39.5 \times 10^{-3}\left( \pm 8.6 \times 10^{-3}\right)$ & $2.0 \times 10^{-3}\left( \pm 0.4 \times 10^{-3}\right)$ & 0.60 \\
\hline $\begin{array}{l}\text { Intracellular trafficking, secretion and } \\
\text { vesicular transport }\end{array}$ & 47 & $66.4 \times 10^{-3}\left( \pm 16.5 \times 10^{-3}\right)$ & $7.0 \times 10^{-3}\left( \pm 3.5 \times 10^{-3}\right)$ & 0.85 \\
\hline Defense mechanisms & 22 & $63.6 \times 10^{-3}\left( \pm 20.1 \times 10^{-3}\right)$ & $3.9 \times 10^{-3}\left( \pm 1.5 \times 10^{-3}\right)$ & 0.76 \\
\hline Not in COGs & 258 & $56.1 \times 10^{-3}\left( \pm 5.9 \times 10^{-3}\right)$ & $6.6 \times 10^{-3}\left( \pm 0.8 \times 10^{-3}\right)$ & 0.72 \\
\hline
\end{tabular}

a Two COG functional categories including one gene (i.e. "RNA processing and modification" and "Extracellular structures") are not showed.

b The average rate $\left(d_{\mathrm{S}}\right)$ of synonymous substitutions (nucleotide mutations that do not alter the amino acid sequence) for all orthologous genes within a give COG role category is displayed, followed by the standard error that is represented in parentheses.

c The average rate $\left(d_{N}\right)$ of nonsynonymous substitutions (nucleotide mutations that alter the amino acid sequence) for all orthologous genes within a give COG role category is displayed, followed by the standard error that is represented in parentheses.

${ }^{\mathrm{d}}$ Correlation coefficient between the values of $d_{\mathrm{S}}$ and $d_{\mathrm{N}}$.

one-sided U-test) and higher nucleotide diversity $(P<$ 0.001 ; one-sided U-test). For all core genome genes, association between COG categories and the number of genes with recombining fragments was estimated (Figure 1). Core genes that exhibit evidence for recombination were significantly overrepresented in three COG categories "Replication, recombination and repair", "Amino acid transport and metabolism", and "Inorganic ion transport and metabolism" (uncorrected $P=0.007, P<0.001$, and $P=0.029$, respectively; one-sided Binomial test). However, after Bonferroni correction, only the association for the COG "Amino acid transport and metabolism" was significant (Bonferroni corrected $P=0.004$ ).

\section{Evidence for 57 A. pleuropneumoniae core genes subject} to positive selection

The analyses of positive selection implemented in PAML was carried out for 1,587 core genome genes of A. pleuropneumoniae (in our initial experiment we included all 1,960 orthologous genes). Based on the LRT statistic for comparing the null model M1a and the selection model M2a with the $\chi_{2}^{2}$ distribution and corrections for multiple testing (FDR $<20 \%$ ), a total of 57 genes were identified to be under strong positive selected pressure (Table 3; Additional file 3). Genes in the COG category "General function prediction only" were significantly enriched $(P=0.004$; one-sided Binomial test). Except for four positively selected genes in the COG category "cell wall/membrane biogenesis", many genes with homologues in other COG categories or without homologues in the COG collection were also predicted to encode proteins localized on surface/membrane and simultaneously subject to positive selective pressure, e.g. gnt T, cys $W, a p a A, p c a K, a p h A, p q i B$ and $y t f$.

Notably, there was no obvious discrepancy for the values of $d_{\mathrm{S}}$ between genes under positive selection and the remaining genes; whereas the $d_{\mathrm{N}}$ values together with the number of informative sites and genetic diversity were significantly higher in the positively selected genes $(P<$ $0.001, P<0.001, P=0.023$; one-sided U-test). No association between positive selection and COG categories was observed, as the number of positively selected genes is low in each role category. 


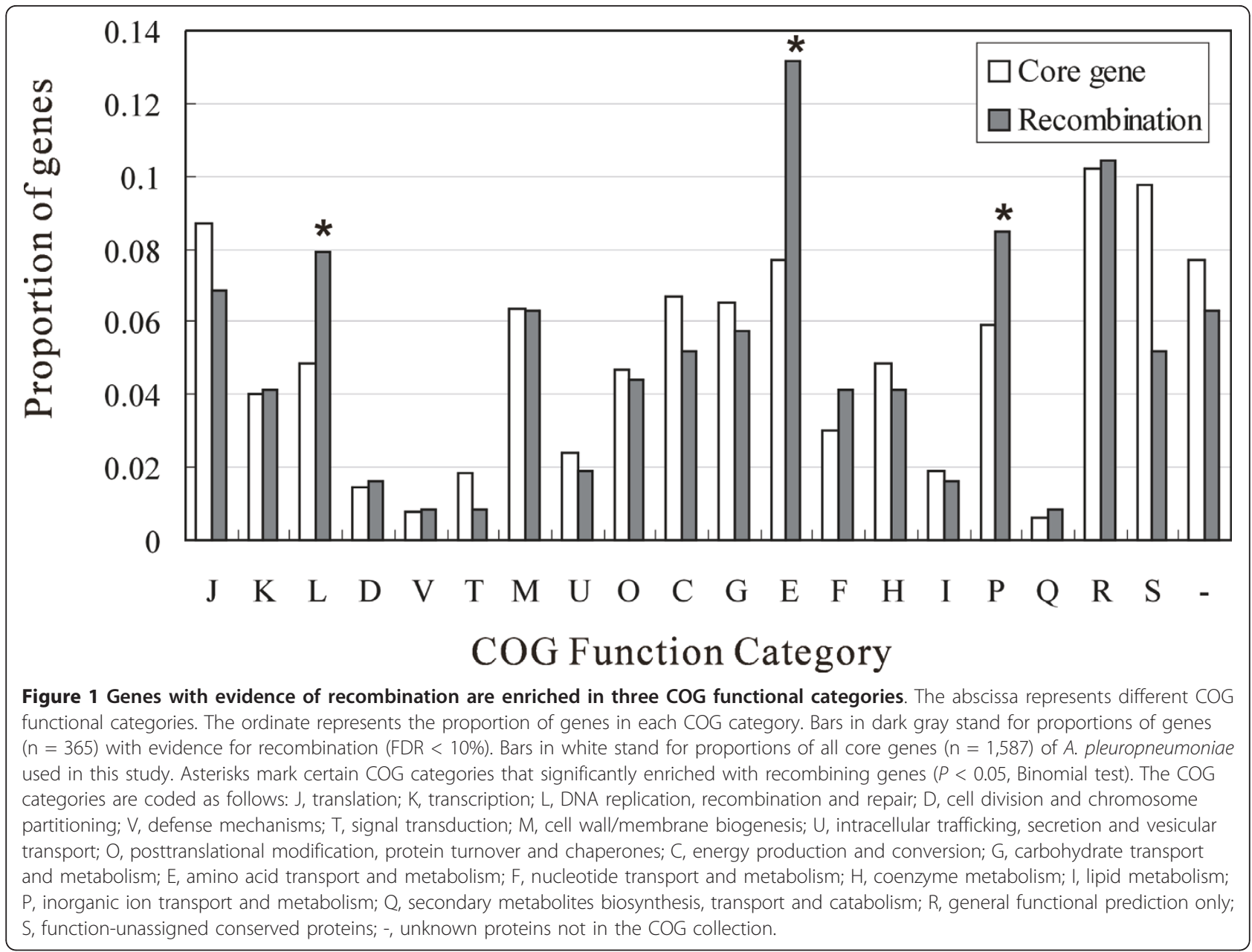

Among 57 positively selected genes, 24 genes also showed significant evidence for homologous recombination detected by all four recombination tests. Furthermore, 41 genes under positive selection pressure showed evidence for recombination identified by at least one test. It indicates that positive selection should be associated with intragenic recombination, as recombination can lead to phylogenic incongruence and highly false positives when selective pressure on protein coding sequences was estimated $[3,29]$.

\section{Discussion}

Gene acquisitions and losses that contribute to the virulence and serotypic diversification of A. pleuropneumoniae have been depicted in detail $[18,19]$, but our understanding on small genetic variations caused by positive selection and homologous recombination, which also factually influence the evolutionary trajectories of protein coding genes, has not been well considered for this swine pathogen so far. In this report, we chose 12 genomes of A. pleuropneumoniae to study the evolutionary driving forces acting on the core genome of this animal pathogen using a comparative phylogenomic approach.

Intragenic recombination and positive selection both play a key role in the evolution of $A$. pleuropneumoniae pangenome

Tests for intragenic homologous recombination and positive selection were performed with 1,587 orthologous genes present in the core genomes of twelve strains of $A$. pleuropneumoniae. Overall, our results indicated that about a quarter of the genes in A. pleuropneumoniae core genome showed significant evidence for intragenic recombination. In comparison, core-genome recombination was also evident in both species of the genus Streptococcus, as $18 \%$ and $37 \%$ of the core genome for S. agalactiae and S. pyogenes, respectively, showed evidence for homologous recombination [5]. Notably, in A. pleuropneumoniae, two COG categories "Replication, recombination and repair" and "Amino acid transport and metabolism", which both presented high values of $d_{\mathrm{S}}$ and $d_{\mathrm{N}}$, were favored by intragenic recombination. 
Table 3 Genes that show evidence for positive Darwinian selection

\begin{tabular}{|c|c|c|c|c|c|c|c|c|}
\hline $\begin{array}{l}\text { Name } \\
\text { (Systematic) }\end{array}$ & $\begin{array}{l}\text { Cluster } \\
\text { ID }^{\mathrm{a}}\end{array}$ & $\mathrm{COG}^{\mathrm{b}}$ & Putative function of coding products & $2 \Delta \ell^{c}$ & $\begin{array}{c}\text { Q- } \\
\text { value }\end{array}$ & $p^{d}$ & $\omega^{\mathrm{e}}$ & $\begin{array}{c}\text { Positively selected } \\
\text { sites } \mathrm{f}\end{array}$ \\
\hline$\overline{\text { napA }}$ & APO_0068 & C & Periplasmic nitrate reductase & 10.03 & 0.189 & 0.000 & 1.02 & \\
\hline$g / p A$ & APO_0197 & C & $\begin{array}{l}\text { Anaerobic glycerol-3-phosphate dehydrogenase } \\
\text { subunit A }\end{array}$ & 12.26 & 0.096 & 0.009 & 11.45 & \\
\hline fumC & APO_0306 & C & Fumarate hydratase & 15.63 & 0.032 & 0.004 & 99.56 & \\
\hline prlc & APO_0109 & E & Oligopeptidase A & 14.72 & 0.042 & 0.000 & 607.84 & \\
\hline $\operatorname{argH}$ & APO_0322 & E & Argininosuccinate lyase & 13.10 & 0.071 & 0.014 & 10.79 & 423 \\
\hline gntT & APO_0415 & E & Transporter, gluconate/H+ symporter (GntP) family & 11.74 & 0.106 & 0.008 & 13.97 & 190, 193 \\
\hline proA & APO_0424 & E & Gamma-glutamyl phosphate reductase & 10.02 & 0.189 & 0.051 & 3.29 & \\
\hline $\operatorname{art} P$ & APO_0998 & E & Arginine transport ATP-binding protein & 14.75 & 0.042 & 0.004 & 521.75 & 225 \\
\hline leuD & APO_1132 & E & 3-isopropylmalate dehydratase small subunit & 11.14 & 0.135 & 0.032 & 57.70 & \\
\hline$c p d B$ & APO_0120 & $\mathrm{F}$ & $\begin{array}{l}\text { 2',3'-cyclic-nucleotide 2'-phosphodiesterase/3'- } \\
\text { nucleotidase }\end{array}$ & 19.13 & 0.010 & 0.032 & 3.82 & $9,99,554$ \\
\hline purD & APO_0394 & $\mathrm{F}$ & Phosphoribosylamine-glycine ligase & 13.44 & 0.062 & 0.061 & 34.74 & \\
\hline$g \lg P$ & APO_00f74 & G & Maltodextrin phosphorylase & 14.13 & 0.054 & 0.019 & 9.66 & $13,27,392$ \\
\hline kdgk & APO_0752 & G & 2-dehydro-3-deoxygluconokinase & 9.98 & 0.190 & 0.042 & 8.28 & \\
\hline hemN & APO_0325 & $\mathrm{H}$ & $\begin{array}{l}\text { Oxygen-independent coproporphyrinogen-III } \\
\text { oxidase }\end{array}$ & 21.87 & 0.005 & 0.015 & 27.37 & $38,136,428$ \\
\hline $\operatorname{lip} B$ & APO_1067 & $\mathrm{H}$ & Octanoyltransferase & 10.14 & 0.189 & 0.000 & 1.00 & \\
\hline vals & APO_0044 & J & Valyl-tRNA synthetase & 22.18 & 0.005 & 0.016 & 3.82 & 47,792 \\
\hline rumA & APO_0356 & J & 235 rRNA (uracil-5-)-methyltransferase & 26.17 & 0.002 & 0.003 & 47.26 & 20 \\
\hline $\operatorname{trmA2}$ & APO_0540 & J & tRNA (uracil-5-)-methyltransferase & 10.70 & 0.160 & 0.028 & 4.08 & 24,210 \\
\hline yhgF & APO_0081 & K & Transcriptional accessory protein & 18.60 & 0.010 & 0.024 & 3.78 & 10,142 \\
\hline lysR & APO_0797 & K & Transcriptional regulatory protein & 18.79 & 0.010 & 0.012 & 10.60 & $284,287,292$ \\
\hline parC & APO_0090 & L & DNA topoisomerase 4 subunit $A$ & 13.47 & 0.062 & 0.003 & 10.50 & 357 \\
\hline mutM & APO_0873 & L & Heptosyltransferase family & 12.15 & 0.096 & 0.077 & 14.90 & 270 \\
\hline tatD & APO_0977 & L & Deoxyribonuclease & 13.73 & 0.059 & 0.198 & 2.42 & $188,204,234$ \\
\hline$h \operatorname{cs} A$ & APO_0102 & M & Capsule polysaccharide modification protein & 10.07 & 0.189 & 0.027 & 3.16 & 483 \\
\hline$h \operatorname{cs} B$ & APO_0367 & M & Capsule polysaccharide modification protein & 18.72 & 0.010 & 0.034 & 18.52 & $13,136,366$ \\
\hline ompP2B & APO_0561 & M & Outer membrane protein P2-like protein & 16.15 & 0.026 & 0.095 & 8.07 & $306,317,320$ \\
\hline msCl & APO_1465 & M & Large-conductance mechanosensitive channel & 12.17 & 0.096 & 0.044 & 103.40 & 97 \\
\hline ptrA & APO_0039 & O & Protease III & 17.69 & 0.013 & 0.049 & 6.26 & $43,44,50,91,617,909$ \\
\hline$g \ln D$ & APO_0064 & O & uridylyltransferase & 17.93 & 0.013 & 0.024 & 12.23 & $17,190,514,551$ \\
\hline sppA & APO_0149 & $\mathrm{O}$ & Protease 4 & 10.42 & 0.173 & 0.030 & 9.37 & \\
\hline IonH & APO_0207 & $\mathrm{O}$ & Lon protease & 15.24 & 0.035 & 0.013 & 106.47 & 19 \\
\hline$n r f G$ & APO_0983 & $\mathrm{O}$ & $\begin{array}{l}\text { Formate-dependent nitrite reductase complex } \\
\text { subunit }\end{array}$ & 12.29 & 0.096 & 0.084 & 27.77 & \\
\hline $\operatorname{teh} A$ & APO_0711 & $\mathrm{P}$ & Tellurite resistance protein & 11.70 & 0.106 & 0.007 & 70.81 & 252 \\
\hline cysW & APO_0883 & $P$ & Sulfate transport system permease protein & 13.80 & 0.059 & 0.074 & 3.68 & 145 \\
\hline- & APO_0030 & $\mathrm{R}$ & Helicase & 12.14 & 0.096 & 0.051 & 2.56 & \\
\hline pqiB & APO_0055 & $\mathrm{R}$ & Paraquat-inducible protein B & 20.31 & 0.008 & 0.046 & 3.62 & $259,318,384$ \\
\hline$t / d D$ & APO_0268 & $\mathrm{R}$ & Protease & 10.16 & 0.189 & 0.009 & 15.91 & 377,379 \\
\hline- & APO_0269 & $\mathrm{R}$ & Hypothetical protein & 10.02 & 0.189 & 0.000 & 1.00 & 463 \\
\hline pcak & APO_0359 & $\mathrm{R}$ & Major facilitator transporter & 12.09 & 0.096 & 0.022 & 5.96 & $195,238,416,417$ \\
\hline thiH & APO_0509 & $\mathrm{R}$ & Thiazole biosynthesis protein & 18.32 & 0.011 & 0.012 & 12.98 & 15,55 \\
\hline murQ & APO_0754 & R & $\mathrm{N}$-acetylmuramic acid 6-phosphate etherase & 17.69 & 0.013 & 0.059 & 4.05 & $54,99,293$ \\
\hline- & APO_0792 & R & Nucleoside-diphosphate sugar epimerase & 11.92 & 0.102 & 0.060 & 9.71 & \\
\hline rSSA & APO_0839 & R & Patatin & 11.22 & 0.132 & 0.019 & 41.55 & 225 \\
\hline cof & APO_0888 & R & Hydrolases of the HAD superfamily & 10.62 & 0.160 & 0.000 & 25.15 & \\
\hline smtA & APO_0969 & $\mathrm{R}$ & Methyltransferase & 10.66 & 0.160 & 0.045 & 101.87 & 134,204 \\
\hline$a p h A$ & APO_1107 & $\mathrm{R}$ & Membrane protein affecting hemolysin expression & 10.83 & 0.153 & 0.012 & 24.96 & 46 \\
\hline rec $X$ & APO_1397 & $\mathrm{R}$ & Regulatory protein & 19.96 & 0.008 & 0.008 & 37.09 & 47 \\
\hline ytfN & APO_0025 & S & Hypothetical protein & 14.07 & 0.054 & 0.002 & 239.60 & 553 \\
\hline
\end{tabular}


Table 3 Genes that show evidence for positive Darwinian selection (Continued)

\begin{tabular}{|c|c|c|c|c|c|c|c|c|}
\hline- & APO_0098 & S & Oligopeptide transporter & 20.79 & 0.007 & 0.012 & 15.91 & $82,127,546$ \\
\hline$g \ln E$ & APO_0041 & $\mathrm{T}$ & Glutamate-ammonia-ligase adenylyltransferase & 11.88 & 0.102 & 0.023 & 3.60 & 53 \\
\hline typA & APO_0150 & $\mathrm{T}$ & GTP-binding protein & 33.59 & 0.000 & 0.006 & 15.43 & $395,567,569$ \\
\hline apaA & APO_0772 & $\mathrm{T}$ & ABC transporter, periplasmic binding protein & 13.49 & 0.062 & 0.031 & 7.10 & 103,147 \\
\hline- & APO_0051 & - & Hypothetical protein & 19.00 & 0.010 & 0.088 & 5.16 & 105 \\
\hline$g u a B$ & APO_0259 & - & Inosine-5'-monophosphate dehydrogenase & 15.25 & 0.035 & 0.013 & 4.98 & 448 \\
\hline- & APO_0393 & - & Hypothetical protein & 24.92 & 0.002 & 0.027 & 57.62 & $97,104,134,355$ \\
\hline- & APO_0571 & - & Hypothetical protein & 12.27 & 0.096 & 0.020 & 28.21 & $199,275,283$ \\
\hline wecF & APO_0577 & - & TDP-Fuc4NAc:lipid II Fuc4NAc transferase & 22.68 & 0.005 & 0.035 & 14.23 & $70,92,182,183,289$ \\
\hline
\end{tabular}

a Protein designations were taken from the A. pleuropneumoniae ortholog annotation that was summarized in our recent publication [19].

b The abbreviations of COG function categories were assigned based on Figure 1.

c $2 \Delta \ell$ denote the statistic of likelihood ratio test.

${ }^{d}$ The proportion of the amino acid sites under positive selection.

e $\omega$ is equal to the ratio of $d_{N}$ to $d_{S}$ (Number of nonsynonymous changes per nonsynonymous sites/Number of synonymous changes per synonymous site) for amino acid sites under positive selection (model M2a).

${ }^{f}$ Positively selected sites identified with posterior probability $P>95 \%$.

On the other hand, 57 A. pleuropneumoniae genes, accounting for approximately $3.6 \%$ of the core genome, were identified to be undergoing positive selection. Another similar study on the identification of genes under positive selection in $E$. coli reported that $0.7 \%$ of 3,505 genes found in six $E$. coli genomes showed evidence for positive selection and no evidence for recombination [1]. Like other pathogenic bacteria, a substantial number of positively selected genes in A. pleuropneumoniae encode protein products involved in the biogenesis and structural components of bacterial cell wall and/or outer membrane. These genes are likely to be associated with co-evolutionary arms races between pathogenic microorganisms and hosts. To further decipher the roles of evolutionary pressure operating on the core genome of A. pleuropneumoniae, we analyzed the functional properties of the positively selected genes and potentially important residues subject to positive selection.

\section{Genes subject to positive selection in $A$. pleuropneumoniae}

We found that many protein products encoded by the positively selected genes were exposed on the cell surface or involved in structural constituents of bacterial cell wall. Some of these proteins have been reported to be important virulence factors associated with bacterial adherence, colonization and persistence. Therefore, it suggests that the genes under diversifying selection may dynamically interact with the host immune and defense systems.

The beta barrel porins are pore proteins that allow the passive diffusion of small, hydrophilic, or changed molecules across Gram-negative bacterial outer membranes [48]. The pore proteins have been believed to be crucial for not only dynamic interactions with the host immune system, but bacterial pathogenesis as well $[1,49]$. An outer membrane protein OmpP2, which was predicted to be beta barrel porin, showed strong evidence for positive selection with a low $q$-value (Table 3 ). The results of the Bayes empirical Bayes (BEB) analyses showed that A. pleuropneumoniae OmpP2 amino acid residues 306, 317 , and 320 were subject to intense positive selective pressure (Figure 2). The three residues all located on a predicted extracellular loop in the $\mathrm{C}$-terminus, perhaps associated with potential antigenic epitope. In addition, OmpP2 has been experimentally confirmed to be essential for in vivo survival of $A$. pleuropneumoniae by signature-tagged mutagenesis and also an immunogenic surface antigen by the immunoproteomic approach [50,51]. In our initial selection analyses using a set of 1,960 genes, gene fepA present in 11 A. pleuropneumoniae genomes encodes a beta barrel porin (Figure 2) and was also identified with evidence for positive selection (data not shown). FepA of A. pleuropneumoniae shared a common TonB-dependent receptor plug domain (PF07715) with E. coli outer membrane protein FepA that is a receptor for ferric enterobactin and for colicins $\mathrm{B}$ and D [52]. FepA of A. pleuropneumoniae has already been reported to exhibit immunogenic activity [53]. The adaptive changes in both porins might be beneficial for A. pleuropneumoniae to escape from the host immune systems and attack of phages, antibiotics, and colicins.

Bacterial surface polysaccharides, which are often involved in adherence and colonization, may be directly exposed to the host immune pressure. Three A. pleuropneumoniae genes ( $h c s A, h c s B$, and wecF) participated in biogenesis of surface polysaccharides showed significant evidence for positive selection. The products of selected genes $h c s A$ and $h c s B$ code for capsule polysaccharide modification proteins that share $63 \%$ and $64 \%$ identity with Haemophilus influenzae HcsA and HcsB, respectively, which facilitate transport of capsular polysaccharide across outer membrane and are essential for bacterial virulence 


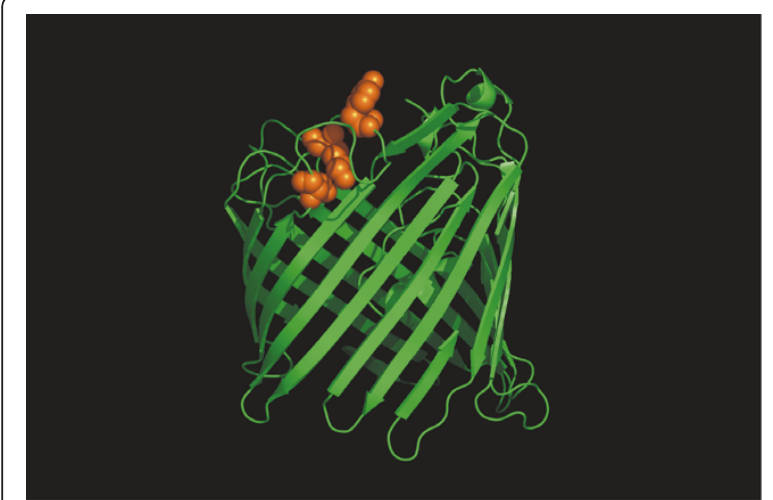

OmpP2

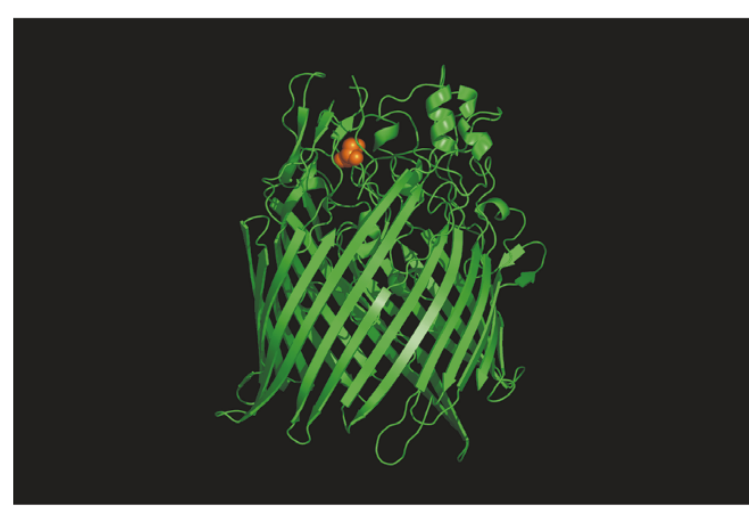

FepA

Figure 2 Three-dimensional structural models of beta barrel porins OmpC and FepA. Orange spheres stand for amino acid sites that are subject to strong positive selection (posterior probability > 95\%).

[54]. Besides, the positively selected gene wecF codes for a 4-alpha-L-fucosyltransferase and is located at a wec locus which has highly conserved colinearity in all A. pleuropneumoniae genomes. The products of wecF together with other wec genes exhibit high similarity to the E. coli $\mathrm{K} 12$ homologues that are involved in the assembly of a cell surface glycolipid [55].

The other gene apaA encoding an antigenic membrane lipoprotein that could provide cross-protection against heterologous A. pleuropneumoniae serovars [56], was also under strong positive selection $(q$-value $=0.062)$. The above analyses strongly demonstrated that the positively selected genes involved in the biosynthesis and structural composition of cell surface/wall have undergone adaptive functional changes, perhaps allowing bacterial pathogens to escape recognition by the host immune system and phages. Such phenomena have already been proposed by the previous studies of natural selection on the E. coli genome $[1,14]$.

The proteases of $A$. pleuropneumoniae have been reviewed to be one of important virulence factors and contribute to pathogenesis [57]. Overall, 4 protease genes (i.e., $p \operatorname{tr} A$, lon $H, s p p A$ and $t l d D$ ) showed significant evidence for positive selection. The precise function of these protease genes identified here, to our knowledge, was not well understood for this pathogen. However, proteolytic enzymes are pivotal to the secretion processes of Gram-negative pathogens and several of them have been described as attractive drug targets in other pathogens, e.g. ClpP [58] and Lon [59].

\section{Conclusion}

Our findings indicated that intragenic homologous recombination and positive Darwinian selection, unsurprisingly, indeed play crucial roles in the evolution of pathogenic A. pleuropneumoniae. In genes with extensive functional classification we found genes involved in the formation of cell surface/membrane are favored by the positive selective pressure. The adaptive changes in these positively selected genes and/or residues likely attribute to dynamic interaction caused by the host immune and defense systems. Of course, the diversifying selective forces of genes encoding metabolic functions may be also advantage for improving bacterial fitness in response to a variety of environmental signals. More experimental works are required for verifying the functions of these adaptive genes in future. Overall, the genetic evidence of positive selection will provide promising targets for further researches in the mechanisms of immune evasion and the host-pathogen interaction in A. pleuropneumoniae.

\section{Additional material}

Additional file 1: Highly conserved genes in the core genome of A. pleuropneumoniae. Detailed information for individual gene alignment is provided, including nucleotide diversity, informative sites, and codon bias.

Additional file 2: Detailed information on test of recombination A. pleuropneumoniae genes showing evidence for recombination detected by at least one method (FDR $<10 \%$ ).

Additional file 3: Alignments for positively selective genes. Compressed file containing all alignments for genes under positive selection (FASTA format).

\section{Acknowledgements}

This work was supported by the National Natural Science Foundation of China (30901075).

\section{Authors' contributions}

ZX carried out the data collection, data analyses, wrote the manuscript. ZX and RZ participated in its design and revised the manuscript. RZ and HC supervised and coordinated the project. All authors read, edited and approved the final manuscript. 


\section{Competing interests}

The authors declare that they have no competing interests.

Received: 9 December 2010 Accepted: 13 July 2011

Published: 13 July 2011

\section{References}

1. Petersen L, Bollback JP, Dimmic M, Hubisz M, Nielsen R: Genes under positive selection in Escherichia coli. Genome Res 2007, 17:1336-1343.

2. Lewis-Rogers N, McClellan DA, Crandall KA: The evolution of foot-andmouth disease virus: impacts of recombination and selection. Infect Genet Evol 2008, 8:786-798.

3. Orsi RH, Sun Q, Wiedmann M: Genome-wide analyses reveal lineage specific contributions of positive selection and recombination to the evolution of Listeria monocytogenes. BMC Evol Biol 2008, 8:233.

4. Soyer Y, Orsi RH, Rodriguez-Rivera LD, Sun Q, Wiedmann M: Genome wide evolutionary analyses reveal serotype specific patterns of positive selection in selected Salmonella serotypes. BMC Evol Biol 2009, 9:264.

5. Lefébure T, Stanhope MJ: Evolution of the core and pan-genome of Streptococcus: positive selection, recombination, and genome composition. Genome Biol 2007, 8:R71.

6. Lefébure T, Stanhope MJ: Pervasive, genome-wide positive selection leading to functional divergence in the bacterial genus Campylobacter. Genome Res 2009, 19:1224-1232.

7. Dawkins R, Krebs JR: Arms races between and within species. Proc $R$ SoC Lond B Biol Sci 1979, 205:489-511.

8. Brunham RC, Plummer FA, Stephens RS: Bacterial antigenic variation, host immune response, and pathogen-host coevolution. Infect Immun 1993, 61:2273-2276.

9. Yang Z, Bielawski JP: Statistical methods for detecting molecular adaptation. Trends Ecol Evol 2000, 15:496-503.

10. Yang Z, Nielsen R, Goldman N, Pedersen AM: Codon-substitution models for heterogeneous selection pressure at amino acid sites. Genetics 2000 155:431-449.

11. Nielsen R, Yang Z: Likelihood models for detecting positively selected amino acid sites and applications to the HIV-1 envelope gene. Genetics 1998, 148:929-936.

12. Golding GB, Dean AM: The structural basis of molecular adaptation. Mol Biol Evol 1998, 15:355-369.

13. Clark AG, Glanowski S, Nielsen R, Thomas PD, Kejariwal A, Todd MA, Tanenbaum DM, Civello D, Lu F, Murphy B, Ferriera S, Wang G, Zheng X, White TJ, Sninsky JJ, Adams MD, Cargill M: Inferring nonneutral evolution from human-chimp-mouse orthologous gene trios. Science 2003, 302:1960-1963.

14. Chen SL, Hung CS, Xu J, Reigstad CS, Magrini V, Sabo A, Blasiar D, Bieri T, Meyer RR, Ozersky P, Armstrong JR, Fulton RS, Latreille JP, Spieth J, Hooton TM, Mardis ER, Hultgren SJ, Gordon Jl: Identification of genes subject to positive selection in uropathogenic strains of Escherichia coli: a comparative genomics approach. Proc Natl Acad Sci USA 2006, 103:5977-5982

15. Frey J: Virulence in Actinobacillus pleuropneumoniae and RTX toxins. Trends Microbiol 1995, 3:257-261.

16. Bossé JT, Janson H, Sheehan BJ, Beddek AJ, Rycroft AN, Kroll JS, Langford PR: Actinobacillus pleuropneumoniae: pathobiology and pathogenesis of infection. Microbes Infect 2002, 4:225-235.

17. Blackall PJ, Klaasen HL, van den Bosch H, Kuhnert P, Frey J: Proposal of a new serovar of Actinobacillus pleuropneumoniae: serovar 15. Vet Microbiol 2002, 84:47-52.

18. Gouré J, Findlay WA, Deslandes V, Bouevitch A, Foote SJ, Maclnnes JI, Coulton JW, Nash JH, Jacques M: Microarray-based comparative genomic profiling of reference strains and selected Canadian field isolates of Actinobacillus pleuropneumoniae. BMC Genomics 2009, 10:88.

19. Xu Z, Chen X, Li L, Li T, Wang S, Chen H, Zhou R: Comparative genomic characterization of Actinobacillus pleuropneumoniae. J Bacteriol 2010, 192:5625-5636

20. Wei W, Cao Z, Zhu YL, Wang X, Ding G, Xu H, Jia P, Qu D, Danchin A, Li Y: Conserved genes in a path from commensalism to pathogenicity: comparative phylogenetic profiles of Staphylococcus epidermidis RP62A and ATCC12228. BMC Genomics 2006, 7:112

21. Holt KE, Parkhill J, Mazzoni CJ, Roumagnac P, Weill FX, Goodhead I, Rance R, Baker S, Maskell DJ, Wain J, Dolecek C, Achtman M, Dougan G: High- throughput sequencing provides insights into genome variation and evolution in Salmonella Typhi. Nat Genet 2008, 40:987-993.

22. Notredame C, Higgins DG, Heringa J: T-Coffee: A novel method for fast and accurate multiple sequence alignment. J Mol Biol 2000, 302:205-217.

23. Kumar S, Nei M, Dudley J, Tamura K: MEGA: a biologist-centric software for evolutionary analysis of DNA and protein sequences. Brief Bioinform 2008, 9:299-306.

24. Nei M, Gojobori T: Simple methods for estimating the numbers of synonymous and nonsynonymous nucleotide substitutions. Mol Biol Evol 1986, 3:418-426.

25. Korber B: HIV Signature and Sequence Variation Analysis. In Computational Analysis of HIV Molecular Sequences. Edited by: Rodrigo AG and Learn GH. Netherlands: Kluwer Academic Publishers; 2000:55-72.

26. Bruen TC, Philippe $H$, Bryant D: A simple and robust statistical test for detecting the presence of recombination. Genetics 2006, 172:2665-2681.

27. Wright F: The 'effective number of codons' used in a gene. Gene 1990, 87:23-29.

28. Peden JF: Analysis of codon usage. PhD thesis University of Nottingham, Department of Genetics; 1999.

29. Anisimova $M$, Nielsen $R$, Yang Z: Effect of recombination on the accuracy of the likelihood method for detecting positive selection at amino acid sites. Genetics 2003, 164:1229-1236.

30. Sawyer S: Statistical tests for detecting gene conversion. Mol Biol Evol 1989, 6:526-538.

31. Smith JM: Analyzing the mosaic structure of genes. J Mol Evol 1992 34:126-129.

32. Jakobsen IB, Easteal S: A program for calculating and displaying compatibility matrices as an aid in determining reticulate evolution in molecular sequence. Comput Appl Biosci 1996, 12:291-295.

33. Guindon S, Gascuel O: A simple, fast, and accurate algorithm to estimate large phylogenies by maximum likelihood. Syst Biol 2003, 52:696-704.

34. Yang Z: PAML 4: phylogenetic analysis by maximum likelihood. Mol Biol Evol 2007, 24:1586-1591.

35. Wong WS, Yang Z, Goldman N, Nielsen R: Accuracy and power of statistical methods for detecting adaptive evolution in protein coding sequences and for identifying positively selected sites. Genetics 2004 168:1041-1051.

36. Yang Z, Wong WS, Nielsen R: Bayes empirical bayes inference of amino acid sites under positive selection. Mol Biol Evol 2005, 22:1107-1118.

37. Yu NY, Wagner JR, Laird MR, Melli G, Rey S, Lo R, Dao P, Sahinalp SC, Ester M, Foster LJ, Brinkman FS: PSORTb 3.0: improved protein subcellular localization prediction with refined localization subcategories and predictive capabilities for all prokaryotes. Bioinformatics 2010, 26:1608-1615.

38. Berven FS, Flikka $K$, Jensen HB, Eidhammer I: BOMP: a program to predict integral beta-barrel outer membrane proteins encoded within genomes of Gram-negative bacteria. Nucleic Acids Res 2004, 32:W394-399.

39. Kelley LA, Sternberg MJ: Protein structure prediction on the Web: a case study using the Phyre server. Nat Protoc 2009, 4:363-371.

40. Benjamini $Y$, Hochberg $Y$ : Controlling the false discovery rate: a practical and powerful approach to multiple testing. J Royal Statis Soc B 1995, 57:289-300.

41. Storey JD, Tibshirani R: Statistical significance for genomewide studies Proc Natl Acad Sci USA 2003, 100:9440-9445.

42. Anisimova M, Bielawski JP, Yang Z: Accuracy and power of the likelihood ratio test in detecting adaptive molecular evolution. Mol Biol Evol 2001, 18:1585-1592

43. R Development Core Team: R: A language and environment for statistical computing: R Foundation for Statistical Computing, Vienna, Austria; 2007 [http://www.R-project.org].

44. Gouy M, Gautier C: Codon usage in bacteria: correlation with gene expressivity. Nucleic Acids Res 1982, 10:7055-7074.

45. Gharbia SE, Williams JC, Andrews DM, Shah HN: Genomic clusters and codon usage in relation to gene expression in oral Gram-negative anaerobes. Anaerobe 1995, 1:239-262.

46. Jordan IK, Rogozin IB, Wolf YI, Koonin EV: Essential genes are more evolutionarily conserved than are nonessential genes in bacteria. Genome Res 2002, 12.962-968.

47. Drummond DA, Bloom JD, Adami C, Wilke CO, Arnold FH: Why highly expressed proteins evolve slowly. Proc Natl Acad Sci USA 2005 102:14338-14343. 
48. Schirmer T: General and specific porins from bacterial outer membranes. J Struct Biol 1998, 121:101-109.

49. Massari P, Ram S, Macleod H, Wetzler LM: The role of porins in neisserial pathogenesis and immunity. Trends Microbiol 2003, 11:87-93.

50. Chung JW, Ng-Thow-Hing C, Budman LI, Gibbs BF, Nash JH, Jacques M, Coulton JW.: Outer membrane proteome of Actinobacillus pleuropneumoniae: LC-MS/MS analyses validate in silico predictions. Proteomics 2007, 7:1854-1865

51. Sheehan BJ, Bossé JT, Beddek AJ, Rycroft AN, Kroll JS, Langford PR: Identification of Actinobacillus pleuropneumoniae genes important for survival during infection in its natural host. Infect Immun 2003, 71:3960-3970.

52. Armstrong SK, Francis $\mathrm{CL}$, Mclntosh MA: Molecular analysis of the Escherichia coli ferric enterobactin receptor FepA. J Biol Chem 1990, 265:14536-14543.

53. Liao Y, Deng J, Zhang A, Zhou M, Hu Y, Chen H, Jin M: Immunoproteomic analysis of outer membrane proteins and extracellular proteins of Actinobacillus pleuropneumoniae JL03 serotype 3. BMC Microbiol 2009, 9:172

54. Sukupolvi-Petty S, Grass S, St Geme JW: The Haemophilus influenzae Type b hcsA and hcsB gene products facilitate transport of capsular polysaccharide across the outer membrane and are essential for virulence. J Bacteriol 2006, 188:3870-3877.

55. Erbel PJ, Barr K, Gao N, Gerwig GJ, Rick PD, Gardner KH: Identification and biosynthesis of cyclic enterobacterial common antigen in Escherichia coli. J Bacteriol 2003, 185:1995-2004

56. Martin PR, Mulks MH: Cloning and characterization of a gene encoding an antigenic membrane protein from Actinobacillus pleuropneumoniae with homology to ABC transporters. FEMS Immunol Med Microbiol 1999, 25:245-254.

57. Chiers K, De Waele T, Pasmans F, Ducatelle R, Haesebrouck F: Virulence factors of Actinobacillus pleuropneumoniae involved in colonization, persistence and induction of lesions in its porcine host. Vet Res 2010, 41:65.

58. Tiwari A, Gupta S, Srivastava S, Srivastava R, Rawat AK: A ClpP protein model as tuberculosis target for screening marine compounds. Bioinformation 2010, 4:405-408.

59. Ingmer $\mathrm{H}$, Brøndsted L: Proteases in bacterial pathogenesis. Res Microbiol 2009, 160:704-710

60. Xu Z, Zhou Y, Li L, Zhou R, Xiao S, Wan Y, Zhang S, Wang K, Li W, Li L, Jin $H$, Kang M, Dalai B, Li T, Liu L, Cheng Y, Zhang L, Xu T, Zheng H, Pu S, Wang B, Gu W, Zhang XL, Zhu GF, Wang S, Zhao GP, Chen H: Genome biology of Actinobacillus pleuropneumoniae JL03, an isolate of serotype 3 prevalent in China. PLoS One 2008, 3:e1450

61. Foote SJ, Bossé JT, Bouevitch AB, Langford PR, Young NM, Nash JH: The complete genome sequence of Actinobacillus pleuropneumoniae L20 (serotype 5b). J Bacteriol 2008, 190:1495-1496.

doi:10.1186/1471-2148-11-203

Cite this article as: Xu et al:: Genome-wide evidence for positive selection and recombination in Actinobacillus pleuropneumoniae. BMC Evolutionary Biology 2011 11:203.

\section{Submit your next manuscript to BioMed Central and take full advantage of:}

- Convenient online submission

- Thorough peer review

- No space constraints or color figure charges

- Immediate publication on acceptance

- Inclusion in PubMed, CAS, Scopus and Google Scholar

- Research which is freely available for redistribution

Submit your manuscript at www.biomedcentral.com/submit
Biomed Central 